\title{
Nearly Isotropic Conjugated Polymer Aggregates with Efficient Local Exciton Diffusion
}

\author{
Youngah Kwon and Laura J. Kaufman* \\ Department of Chemistry, Columbia University, New York, NY 10027, United States \\ *corresponding author: kaufman@,chem.columbia.edu
}

\section{Supporting Information}

\section{Supporting Text}

\section{Calculation of Number of Single Molecules per Aggregate, $N_{S M-A G G}$}

The number of single molecules per aggregate, $N_{\mathrm{SM}-\mathrm{AGG}}$ was calculated via

$$
N_{S M-A G}=\frac{\frac{1}{I_{S M, B}}\left\{I_{\text {film }, B}-\frac{1}{C}\left(I_{\text {film }, A}-\sum_{1}^{N_{A G G}} I_{A G G}\right)\right\}}{N_{A G G}}
$$

Here, $N_{\mathrm{AGG}}$ is the number of aggregates within the imaging area, $I_{\mathrm{SM}, \mathrm{B}}$ is the median fluorescence intensity of a single chain before SVA, $I_{\text {film }, \mathrm{B}}$ is the total intensity of the fluorescence image before SVA, $I_{\text {film,A }}$ is the total intensity of the fluorescence image after SVA, $\Sigma I_{\mathrm{AGG}}$ is the sum of fluorescence intensities from aggregates within an imaging area. $C$ is a correction factor that was used previously to account for decreased fluorescence intensity from the background of films after solvent vapor annealing. ${ }^{1}$ In our previous publication on aggregates formed from MEH-PPV initially dissolved in toluene, the decrease was attributed to enhanced compaction of single molecules remaining in the background and contributing to total film intensity after SVA. Here, given the set of findings described in the main text, this correction factor is obtained from concentration A and accounts for the presence of small aggregates in the background of films $\mathrm{C}$ and $\mathrm{D}$ following SVA. $C$ is set to 1 for aggregate $\mathrm{B}$ and is set to 0.82 for aggregates $\mathrm{C}$ and $\mathrm{D}$, with this number obtained from the median intensity of features in films of concentration A before and after SVA, assuming two molecules per aggregate. We note that 0.82 may be an overestimate of the correction factor if there is more aggregation and quenching in the background of films $\mathrm{C}$ and $\mathrm{D}$ than that which occurs in films of concentration $\mathrm{A}$, which in turn would lead to an overestimate of the number of chains per aggregate in concentrations $\mathrm{C}$ and $\mathrm{D}$. We thus consider the numbers presented in the main text to be upper bounds on the number of chains per aggregate. 


\section{Supporting Figures}

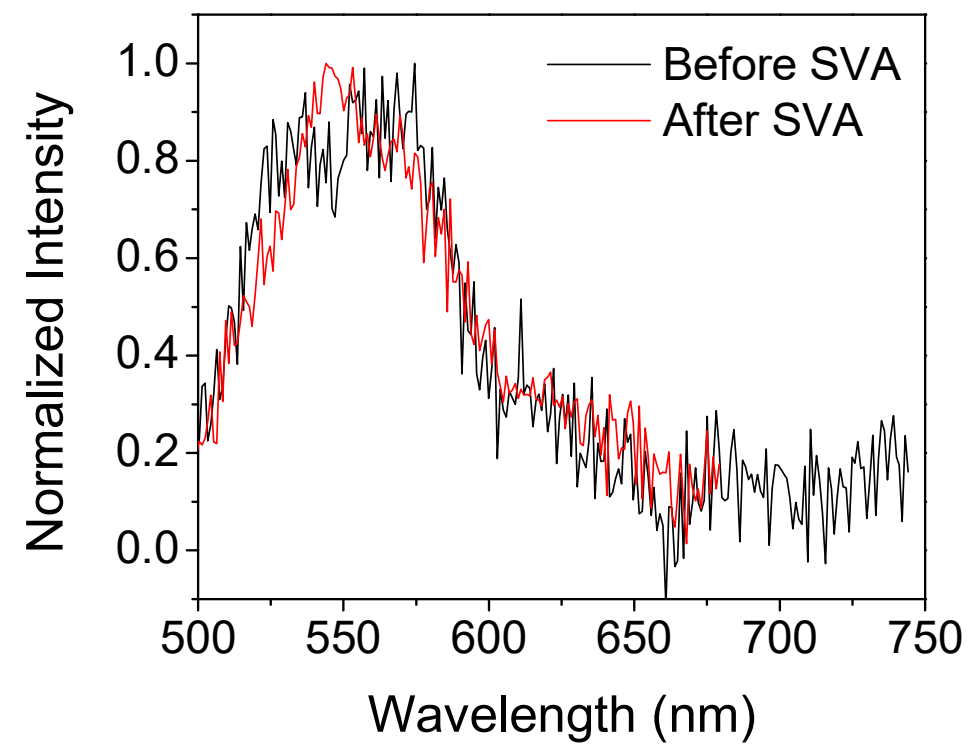

Figure S1. Average spectra of features identified in films of concentration A (black) before and (red) after SVA with swelling performed to a lesser degree and for a shorter time than in other measurements, as described in the main text. These spectra were collected using a transmission grating in front of a CCD camera rather than on a spectrograph as described in the Methods section and as used for collecting spectra presented in Figs. 5 and 6 in the main text. Spectra were averaged over 10 and 15 individual features before and after SVA, respectively, and the excitation power density was $300 \mathrm{~W} / \mathrm{cm}^{2}$.

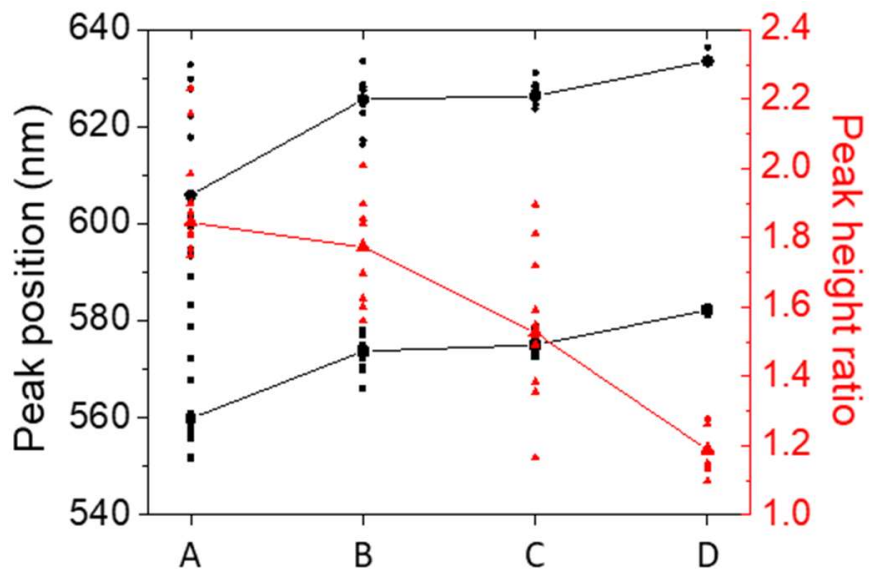

Figure S2. (a) Measured (black squares) 0-0 peak position, (black circles) 0-1 peak position, and (red triangles) $0-0 / 0-1$ peak height ratios for features in films of concentration A - D after SVA. Each spectrum was fit as described in the main text. Large symbols represent median values and small symbols are those for each of $\mathrm{n}=12,9,12$, and 7 features for $\mathrm{A}-\mathrm{D}$, respectively, that contribute to the data shown in Fig. 6 in the main text. 

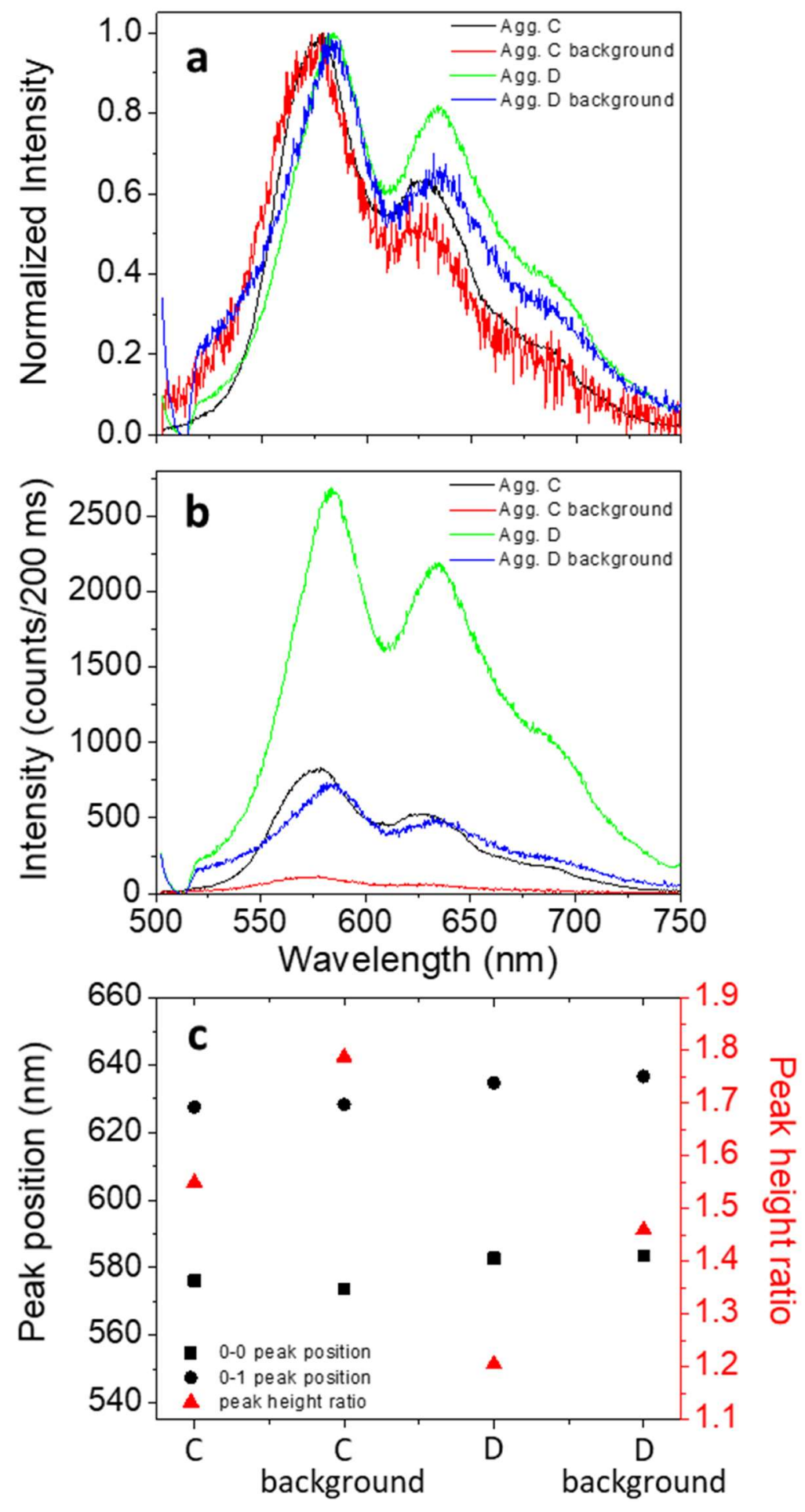

Figure S3. (a) Normalized average spectra of aggregates C and D together with spectra collected from the background between features following SVA. The excitation power densities were $330 \mathrm{~W} / \mathrm{cm}^{2}$ for aggregates $\mathrm{C}$ and their background and $88.7 \mathrm{~W} / \mathrm{cm}^{2}$ for aggregates $\mathrm{D}$ and their background. (b) The same spectra as (a) but without normalization. (c) (Black squares) 0-0 and (black circles) 0-1 peak positions and (red triangles) $0-0 / 0-1$ peak height ratios of aggregates $\mathrm{C}$ and $\mathrm{D}$ and their backgrounds obtained from spectra shown above. 


\section{Reference}

(1) Yang, J.; Park, H.; Kaufman, L. J. Highly Anisotropic Conjugated Polymer Aggregates:

Preparation and Quantification of Physical and Optical Anisotropy. J. Phys. Chem. C 2017, 121, 13854-13862. 